\title{
ARTICLE OPEN The origin of Mooij correlations in disordered metals
}

\author{
Sergio Ciuchi ${ }^{1,2}$, Domenico Di Sante ${ }^{3}$, Vladimir Dobrosavljević ${ }^{4}$ and Simone Fratini $\mathbb{1}^{5}$
}

Sufficiently disordered metals display systematic deviations from the behavior predicted by semi-classical Boltzmann transport theory. Here the scattering events from impurities or thermal excitations can no longer be considered as additive-independent processes, as asserted by Matthiessen's rule following from this picture. In the intermediate region between the regime of good conduction and that of insulation, one typically finds a change of sign of the temperature coefficient of resistivity, even at elevated temperature spanning ambient conditions, a phenomenology that was first identified by Mooij in 1973. Traditional weak coupling approaches to identify relevant corrections to the Boltzmann picture focused on long-distance interference effects such as "weak localization", which are especially important in low dimensions (1D and 2D) and close to the zero-temperature limit. Here we formulate a strong-coupling approach to tackle the interplay of strong disorder and lattice deformations (phonons) in bulk three-dimensional metals at high temperatures. We identify a polaronic mechanism of strong disorder renormalization, which describes how a lattice locally responds to the relevant impurity potential. This mechanism, which quantitatively captures the Mooij regime, is physically distinct and unrelated to Anderson localization, but realizes early seminal ideas of Anderson himself, concerning the interplay of disorder and lattice deformations.

npj Quantum Materials (2018)3:44; doi:10.1038/s41535-018-0119-y

\section{INTRODUCTION}

Identifying physical mechanisms that control electronic transport in materials have long been recognized as a central task in condensed matter physics. In good conductors, for example, it is known that finite electrical resistivity arises due to scattering processes from impurities or various thermal excitations, as very successfully described by the Boltzmann theory of transport. Here, the various scattering events can be considered as statistically independent and thus additive, leading to the well-known Matthiessen's rule, where any thermally induced scattering simply increases the resistivity $\rho(T)$. This behavior, which is well documented in most metals, corresponds to a positive temperature coefficient of resistivity (TCR), i.e., $\mathrm{d} \rho / \mathrm{d} T>0$.

At stronger disorder, where the residual resistivity $\rho_{0}=\rho(T=0)$ increases toward the Mott-loffe-Regel (MIR) limit ${ }^{1-4}$ (corresponding to short scattering lengths on the atomic scale), significant deviations to the weak scattering picture are commonly observed, eventually leading to a change of sign of the TCR. ${ }^{5,6}$ One popular view on the possible cause for this behavior ${ }^{7-10}$ invokes a mechanism for impurity-induced bound electronic state formation at strong disorder-the so-called Anderson localization. ${ }^{11}$ This picture involves a modification of the electronic wavefunctions due to interference processes, but it does not envision any significant rearrangement of the electronic spectra-no gap needs to open. The related "weak localization" corrections prove to describe well the leading low-temperature behavior in metals at weak disorder, where careful perturbative treatments have provided a consistent theoretical picture. This is the regime where such interference processes dominate, but it is by no means obvious that they do so at stronger disorder or around room temperature.

On the other hand, a surprisingly robust phenomenology describing how the TCR changes sign around the MIR limit has been established by Mooij in the early $1970 s^{5}$ noticing that the slope of the resistivity curves linearly (anti)correlates with the extrapolated zero-temperature value $\rho_{0}$, which has subsequently been confirmed on hundreds of materials (i.e., essentially every metal where a sufficient degree of disorder could be experimentally achieved). ${ }^{2,6,8,12,13}$ Most remarkably, such apparent universality was found in the high-temperature regime, often extending to hundreds of Kelvin. This observation is important, since nonlocal interference processes underpinning (weak) localization require long-distance phase coherence, a situation that can hardly be expected to hold at elevated temperatures where incoherent thermal excitations abound. Indeed, the transport behavior of metals in this temperature regime is known to be dominated by lattice vibrations (phonons), leading to the familiar linear-T resistivity above the Debye temperature. The aim of this work is to propose, and validate against available experiments, an alternative scenario that can more plausibly explain the ubiquitous high-temperature anomalies identified by Mooij.

To set the stage for our approach, we recall that alternative physical ideas have been put forward in early work by Anderson himself, in discussing the possible role of lattice deformations in the limit of strong disorder. ${ }^{14} \mathrm{He}$ argued that the reduced mobility of electrons in poor conductors may allow the lattice deformations to self-trap electrons through a disorder-assisted polaronic effect. As in other examples of strong interaction effects, this polaronic mechanism should result in rearrangements of the electronic energy levels, leading to gap formation and substantial transfer of spectral weight away from the Fermi energy. In recent work ${ }^{15}$ we formulated a microscopic theory able to capture both Anderson localization and such strong-coupling polaronic effects. Importantly, it was shown that the latter always acquire a dominant role at strong disorder (i.e., surprisingly, polaronic deformations

\footnotetext{
${ }^{1}$ Department of Physical and Chemical Sciences, University of L'Aquila, Via Vetoio, I-67100 L'Aquila, Italy; ${ }^{2}$ Consiglio Nazionale delle Ricerche (CNR-ISC), Via dei Taurini, I-00185

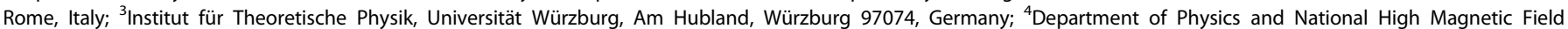
Laboratory, Florida State University, Tallahassee, FL 32306, USA and ${ }^{5}$ Univ. Grenoble Alpes, CNRS, Grenoble INP, Institut Néel, 38000 Grenoble, France Correspondence: Simone Fratini (simone.fratini@neel.cnrs.fr)
}

Received: 16 March 2018 Revised: 27 July 2018 Accepted: 22 August 2018

Published online: 13 September 2018 

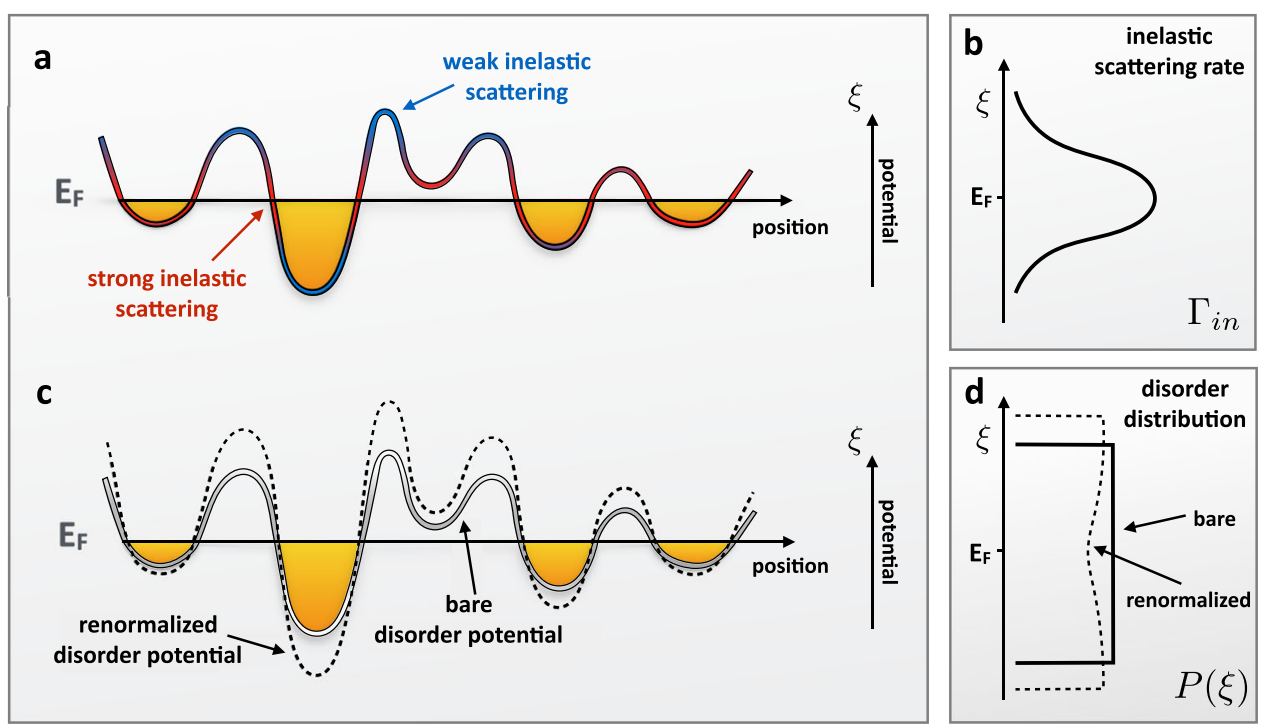

Fig. 1 Breakdown of Matthiessen rule and polaronic renormalization of disorder. $\mathbf{a}, \mathbf{b}$ The rate of inelastic scattering correlates with the value of the local random potential $\xi$, being maximum at the Fermi energy $E_{\mathrm{F}}$ and minimum away from it, as indicated by the color code (red: strong scattering, blue: weak scattering). c, d The deformable lattice responds to the spatial fluctuations of the electron density (shaded yellow), renormalizing the random potential (dashed line), which opens a dip in the distribution $P(\xi)$

naturally arise even in common metals with weak electron-phonon interactions), provided that one releases the customary assumption where metals are regarded as infinitely rigid, undeformable objects. 6,11 The main physical point of the present paper is to emphasize that this mechanism, while completely unrelated to Anderson localization, holds the key to understanding the universal high-temperature anomalies in the Mooij regime. We do this by formulating a theory which by construction disregards non-local interference effects associated with Anderson localization, but does capture strong-coupling physics associated with disorder-assisted polaron formation. We show that important local correlations between the impurity potential and the induced lattice deformations directly cause the breakdown of Matthiessen's rule, and in fact allow quantitative description of Mooij correlations found around the MIR limit.

We intend to address the deviations from Matthiessen's rule observed in sufficiently disordered metals at high temperatures, and to demonstrate the origin of the existing relation between the temperature coefficient of the resistivity, $d \rho / \mathrm{d} T$, and its residual zero-temperature value $\rho_{0}$. To this end we apply dynamical mean field theory in the coherent potential approximation (DMFT-CPA)an inherently strong-coupling theory which takes the viewpoint that the dominant physical processes occur at the local level, and allows us to include both disorder and the desired interaction effects in a reliable way ${ }^{16}$ (see the SM file, Sec. 1). The temperaturedependent resistivity is then evaluated using the Kubo-Greenwood formula, i.e., explicitly going beyond the semi-classical Boltzmann treatment. We provide next an analytical derivation illustrating the physical origin of the phenomenon in full generality, and then proceed with the discussion of the numerical DMFT-CPA results.

\section{RESULTS}

Breakdown of Matthiessen's rule and Mooij correlations

Let us consider a disordered metal at $T=0$, which we take as our reference system. In the local picture, the solution of the disorder problem is entirely characterized by the local Green's function (or, equivalently, the local density of states). This quantity, that we denote by $\hat{G}_{e l}^{\xi}(\omega)$, varies from site to site depending on the value of the local random potential $\xi$, and is entirely determined from the knowledge of the statistical distribution $P(\zeta)$ of site energies.
From its averaged value over the sample, $G_{e l}(\omega)=\left\langle\hat{G}_{e l}^{\xi}(\omega)\right\rangle$, one determines the self-energy $\Sigma_{e l}(\omega)$, which incorporates the relevant elastic scattering processes related to the random environment. The residual resistivity $\rho_{0}$ of the metal at $T=0$ is then readily evaluated from the elastic scattering rate $\Gamma_{e l}=-2 \operatorname{lm} \Sigma_{e l}\left(\omega=E_{\mathrm{F}}\right)$, with $\rho_{0} \propto \Gamma_{e l}$ from the Drude theory of metals.

The temperature coefficient of the resistivity can now be addressed by performing an expansion in the lattice fluctuations, as these are responsible for the leading $\rho \propto T$ term (thermal smearing of the Fermi surface is negligible at this stage, as it brings corrections $\propto T^{2}$ and therefore does not affect the TCR, see Fig. S2 in the SM file). Using the Dyson equation, we obtain the total self-energy, which incorporates both elastic and inelastic scattering, as:

$\Sigma=\Sigma_{e l}+G_{e l}^{-1}\left\langle\hat{G}_{e l}^{\xi} \hat{\Sigma}_{i n}^{\xi} \hat{G}_{e l}^{\xi}\right\rangle G_{e l}^{-1}$.

(all quantities here and in what follows are functions of frequency $\omega$, which we omit for clarity). The term $\hat{\Sigma}_{i n}^{\xi}$ describes the inelastic emission and absorption of phonons. Its explicit dependence on the atomic site energy $\xi$ indicates that the way an electron is affected by such inelastic processes is different from site to site, depending on its local random environment, as sketched in Fig. $1 \mathrm{a}, \mathrm{b}$. Equation (1) reveals how correlations between disorder and electron-phonon scattering emerge: the propagation in the disordered lattice and the interaction processes are intertwined because they take place in the same region of the experimental sample, symbolized here by the same given value of the sitedisorder variable $\xi$. The formal separation between scattering channels underlying Matthiessen's rule would arise only if we were to treat the different terms in Eq. (1) as independent processes, which corresponds to factorizing the averages as $\left\langle\hat{G}_{e l}^{\xi}\right\rangle\left\langle\hat{\Sigma}_{\text {in }}^{\xi}\right\rangle\left\langle\hat{G}_{\text {el }}^{\xi}\right\rangle$ : this would yield $\Sigma=\Sigma_{e l}+\Sigma_{\text {in }}$ with $\Sigma_{\text {in }}=\left\langle\hat{\Sigma}_{\text {in }}^{\xi}\right\rangle$, and the scattering rate $\Gamma=-2 \operatorname{lm} \Sigma$ would indeed separate into a sum of two contributions from the phonons and from disorder, respectively, $\Gamma=\Gamma_{e l}+\Gamma_{i n}$. In general, however, one must use the fully disorder-dependent scattering rate, that we write here as

$\Gamma=\Gamma_{e l}+\Phi \Gamma_{i n}$,

having defined the dimensionless vertex function $\Phi=\frac{\left\langle\hat{G}_{e l}^{\xi} \hat{\Sigma}_{i \text { i }}^{\xi} \hat{G}_{e l}^{\xi}\right\rangle}{\left\langle\hat{G}_{e l}^{\xi}\right\rangle^{2}\left\langle\hat{\Sigma}_{i n}^{\xi}\right\rangle^{\prime}}$ 
which embodies lattice/disorder correlations. Explicit deviations from Matthiessen's rule arise as soon as $\Phi<1$. It should be stressed that we are implicitly ignoring here additional deviations that can arise when the self-energy has a non-trivial matrix structure (due, e.g., to non-local effects, not included in Eq. (1), or to multi-band effects beyond our simple band model).

The lowest-order inelastic contribution (phonon exchange) entering in Eq. (1) is readily obtained as $\hat{\Sigma}_{i n}^{\xi}=s^{2} \hat{G}_{e l}^{\xi}$, highlighting the dependence of inelastic scattering on the local environment variables. The prefactor $s^{2}$ measures the fluctuations of the site energy induced by the atomic motions. These motions are thermal above the Debye temperature, leading to $s^{2} \propto T$ owing to the equipartition principle, which ultimately causes a linear resistivity $\rho \propto \Gamma_{\text {in }} \propto T$ (an explicit calculation assuming a local interaction with Einstein phonons is provided in the SM file, p.11). The above expression for $\hat{\Sigma}_{i n}^{\xi}$, together with Eq. (2), allows us to explicitly relate the TCR to the properties of the disordered metal at $T=0$. In particular, neglecting the weak temperature dependence of $\Gamma_{e l}$ in Eq. (2) we obtain:

$\frac{d \Gamma}{d T} \simeq \frac{d \Gamma_{\text {in }}}{d T} \times \Phi$.

This expression is very appealing because it separates the temperature dependence of the resistivity into a conventional term $\frac{d \Gamma_{\text {in }}}{d T}>0$ connected with the inelastic scattering off the phonons in the absence of disorder, and a factor $\Phi=$ $\left\langle\left(\hat{G}_{e l}^{\xi}\right)^{3}\right\rangle /\left\langle\hat{G}_{e l}^{\xi}\right\rangle^{3}$ controlled by disorder alone, and which is entirely responsible for the sign of $\mathrm{d} \Gamma / \mathrm{d} T$.

To demonstrate the emergence of Mooij correlations, we now expand Eq. (3) in the weak disorder limit. Both $\Phi$ and $\Gamma_{e l}$ can be explicitly evaluated by expressing the local Green's function $\hat{G}_{e l}^{\xi}=$ $\left(G_{0}^{-1}-\xi\right)^{-1}$ in terms of the Weiss field $G_{0}^{-1}$, which embodies the propagation from a given site to the rest of the lattice. Having checked numerically that the results do not change qualitatively as the band filling is varied within the metallic phase, we present here the particle-hole symmetric case for simplicity. In this case, $G_{0}^{-1}=i \nu$ is purely imaginary, where $v$ defines the escape rate from an atomic site, which leads to $\Phi \simeq 1-3 \Gamma_{e l} / 2 v$ for $\Gamma_{e l} \ll \nu$, with $\Gamma_{e l}=$ $2\left\langle\xi^{2}\right\rangle / v$. The above expansion is totally general: when combined with Eq. (3), it states that the temperature dependence of the scattering rate in a weakly disordered metal is entirely known in terms of its behavior in the clean limit and of the residual zerotemperature value $\Gamma_{e l}$. Moreover, the relationship is governed by a single number $v$ which is proportional to the average density of states (DOS) at the Fermi energy (from the Fermi golden rule, $v \sim$ $t^{2} N\left(E_{F}\right) \sim t^{2} / t$ with $t$ the inter-atomic transfer integral). Substituting into Eq. (3) and using the Drude formula to convert to resistivities directly implies

$\rho(T)=\rho_{0}+\left(\rho^{*}-\rho_{0}\right) A T$,

with $A$ and $\rho^{*}$ material-specific constants. In the weak disorder limit where the above formula applies, $\rho \lesssim \rho^{*}$, the TCR is indeed linearly anti-correlated with the residual value $\rho_{0}$.

The foregoing derivation leads us to the following important conclusions. First, it demonstrates that neither the Mooij correlations nor the existence of an anomalous metallic regime with $\frac{d \rho}{d T}<0$ require to be in the immediate proximity to a metal-insulator transition. This is further confirmed by the fact that accounting for Anderson localization using the more general method of ref. ${ }^{15}$ does not alter the overall picture described here (cf. Fig. S4 in the SM file). Quite on the opposite, the Mooij phenomenon is rooted in those correlations between inelastic and elastic scattering that arise already at weak disorder, and which are also responsible for the breakdown of Matthiessen's rule. Second, in those cases where the decrease predicted by Eq. (4) extends all the way down to the point where the slope $\frac{\mathrm{d} \rho}{\mathrm{d} T}$ changes sign, then the $T$ independent resistivity is predicted to occur when the scattering rate is of the order of a fraction of the escape rate. Since this is also a fraction of the electronic bandwidth, the above argument shows that the condition for flat resistivity and the MIR limit qualitatively coincide, as was long assumed.

Polaronic renormalization of disorder

The occurrence of an anomalous metallic regime where $\mathrm{d} \rho / \mathrm{d} T$ changes sign sets precise conditions on the form of the actual disorder distribution $P(\xi)$. From the general expression of $\Phi \propto$ $-\int d \xi \frac{1}{\nu^{2}+\xi^{2}} \frac{d^{2} P}{d \xi^{2}}$ (Eq. (S19) in the SM file), it is apparent that for sufficiently strong disorder, i.e., when the fluctuations of the site energies $\xi$ become large on the scale of the escape rate $v$, the sign of the correlation vertex $\Phi$ is fully determined by the curvature of $P(\xi)$ around $\xi=0$. Given that $\mathrm{d} \rho / \mathrm{d} T \propto \Phi$, a sufficient condition for the emergence of negative TCR is therefore the existence of a dip in the disorder distribution around the Fermi energy. Conversely, according to this same argument a featureless distribution of site energies as is commonly assumed in theoretical studies of disordered systems ${ }^{11}$ is unable to yield such a change of sign, because $\frac{d^{2} P}{d \xi^{2}}=0$. Similarly, no anomalous temperature dependence should arise in the case of gaussianly distributed site energies $\left(\frac{d^{2} p}{d \xi^{2}}<0\right)$, as realized for example in systems hosting randomly distributed charges or dipoles. ${ }^{17}$ The dimensionless function $\Phi\left(\Gamma_{e l} / v\right)$ for different distributions of disorder is illustrated in Fig. S1 of the SM file.

The prediction of a large variability of behaviors depending on the form of disorder seems at odds with the general experimental observation of resistivities with negative temperature coefficients in sufficiently disordered metals. The origin of this widespread behavior lies in the response of the deformable lattice, which is able to modify the very nature of elastic scattering (Fig. 1c): triggered by the existing randomness, polaronic deformations will inevitably arise and convert any given, even featureless, disorder distribution into one with the required characteristic dip (Fig. 1d). ${ }^{15}$ Furthermore, such polaronic renormalization of the disorder potential is not restricted to materials with particularly strong electron-phonon interactions but is expected to occur in all metals, even those which have nominally weak interactions. This can be understood as follows. In the presence of a non-vanishing electron-phonon coupling, the lattice locally distorts in response to the inhomogeneities of the electron liquid. This gives rise to a self-consistent (Hartree) potential $\Sigma^{H}(\epsilon)$, which adds to the random site potential $\epsilon$, so that the total potential felt by the electrons is now the sum $\xi=\epsilon+\Sigma^{H}(\epsilon)$. The induced potential behaves exactly as the induced magnetization in an applied external magnetic field, being $\Sigma^{H}(\epsilon) \propto \operatorname{sign}(\epsilon)$ at large $\epsilon::^{15}$ it is attractive for sites whose energy is below the Fermi level, and repulsive above the Fermi level, so that it effectively shifts the site energies away from $\epsilon=0$, separating the lattice sites into those which host a polaron and those which do not. As a result, upon inclusion of the response of the deformable lattice, the actual distribution $P(\xi)$ of the renormalized local potentials inevitably develops a dip around $\xi=0$ (Fig. 1d). According to the general arguments given in the preceding paragraph, this eventually enables $\mathrm{d} \rho / \mathrm{d} T<0$ regardless of the shape of the initial distribution of $\epsilon$. We stress that the inclusion of dephasing or inelastic scattering effects which do not affect the real part of the self-energy are instead unable to modify the effective disorder distribution. ${ }^{18,19}$

Numerical results

Having illustrated the physical mechanisms at play in full generality, we now proceed by studying two representative 
a

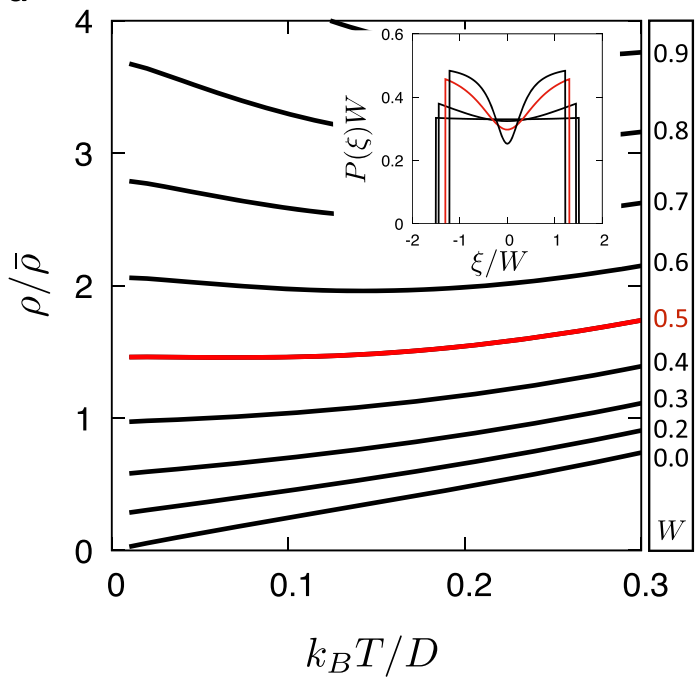

b

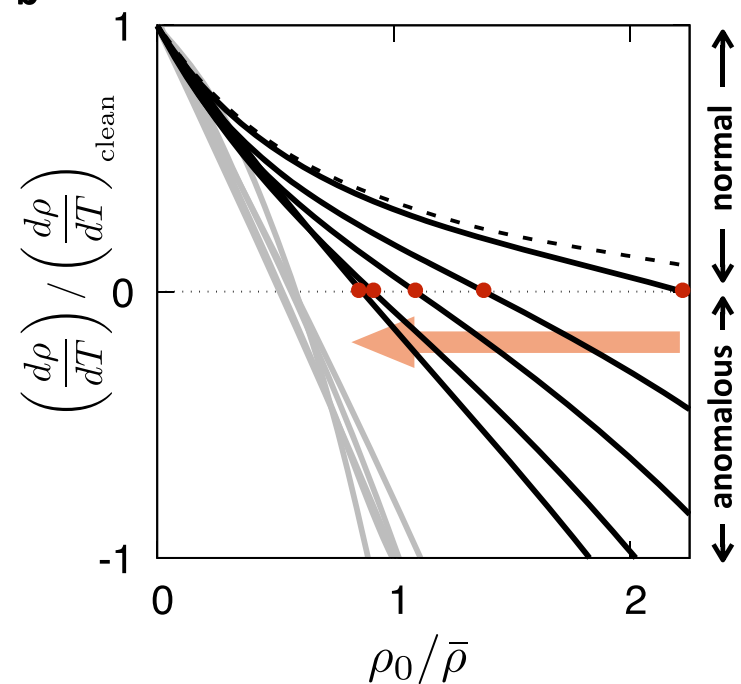

Fig. 2 Numerical results. a Resistivity vs. temperature in the uniform disorder model at fixed $\lambda=0.2$, for increasing disorder strengths $W$ as indicated by the labels (temperature and $\rho$ units are set respectively by the half-bandwidth $D$ and $\bar{\rho}=a \hbar / e^{2}$ ). The flat resistivity curve is marked in red. Inset: The corresponding renormalized site energy distribution $P(\xi)$ at $T=0$, for selected values of $W=0.0$ (bare distribution, see text), $0.2,0.5,0.8$. b The rate of variation $\mathrm{d} \rho / \mathrm{d} T$ extracted from the resistivity data in the linear range $k_{\mathrm{B}} T / \mathrm{D}=0.01-0.05$ normalized by its value in the clean limit as a function of $\rho_{0} / \bar{\rho}$, for the uniform disorder model (black lines). The different curves represent increasing values of $\lambda$ $=0.05,0.2,0.3,0.4,0.5$, in the order indicated by the arrow, spanning essentially the entire metallic region allowed by the theory (see text). The dashed line indicates the $\lambda \rightarrow 0$ limit. Red dots mark the locus $\rho^{*}$ of flat resistivity. The results for the binary disorder model, shown as gray lines, do not exhibit appreciable $\lambda$-dependence in the region under study

models for disordered metals using the fully self-consistent DMFTCPA method. Figure $2 \mathrm{a}$ reports $\rho(T)$ calculated considering a uniform initial distribution of site energies $P_{0}(\epsilon)=\theta\left(W^{2}-\epsilon^{2}\right) /(2 W)$ for a generic metal characterized by a featureless semi-elliptical DOS of half-width $D$ and an electron-phonon interaction of moderate strength, $\lambda=0.2$ (we consider here an onsite interaction with dispersionless phonons characterized by a force constant $K$ and coupling constant $g$, so that $\lambda=g^{2} /(K D)$, see Eq. (S1) in the SM file for details). We conveniently express the resistivity in units of $\bar{\rho}=a \hbar / e^{2}\left(\sim 10^{2} \mu \Omega \mathrm{cm}\right.$ for typical simple metals, assuming a typical lattice spacing $a \simeq 3 \AA$ ), which is of the order of the MIR limit. The resistivity curves reproduce the typical phenomenology observed in experiments, both qualitatively and quantitatively. ${ }^{6}$ In particular, the flat resistivity (red curve) occurs for values close to $\bar{\rho}$ as anticipated. The evolution of the TCR as a function of the zerotemperature intercept $\rho_{0}$ is reported in Fig. 2b (black full lines, different curves corresponding to different values of $\lambda$ ). At weak disorder, all curves do tend to a common linear behavior as predicted by our analytical derivation.

We now explore the dependence on the electron-phonon interaction strength, spanning the whole metallic regime up to the breakdown of the metal, which is delimited at large $\lambda$ by the formation of a polaronic insulator (the critical value for the polaronic transition here is $\lambda_{\mathrm{p}}=0.67$ in the clean limit, ${ }^{20}$ which is progressively reduced by the inclusion of disorder ${ }^{15}$ ). The black dashed curve is the limiting behavior obtained in the uniform disorder model for vanishingly weak electron-phonon interactions, $\lambda \rightarrow 0$. In the absence of electron-phonon interactions, the flat distribution $P_{0}(\epsilon)$ does not allow for negative values of $\mathrm{d} \rho / \mathrm{d} T$, so that the initial decrease at low $\rho_{0}$ progressively flattens out and saturates for strong scattering. The situation changes radically as soon as $\lambda \neq 0$. As shown in the inset of Fig. 2a, the distribution $P(\xi)$ is progressively depleted at $\xi \simeq 0$ owing to the buildup of polaronic lattice deformations; this enables $\mathrm{d} \rho / \mathrm{d} T<0$ above a finite $\rho^{*}$, precisely as demonstrated in the preceding paragraphs. We see from Fig. $2 b$ that the polaronic effects responsible for the anomalous metallic behavior are crucial already for quite modest interaction strengths, typical of metals: the locus $\rho^{*}$ of flat resistivity (red dots), which diverges for $\lambda \rightarrow 0$, rapidly decreases with $\lambda$ and reaches values of the order of the resistivity unit $\bar{\rho}$ already for $\lambda=0.05$. This demonstrates that, aside from specific cases where the electron-phonon coupling is particularly weak, the flat resistivity can generally be identified with the MIR limit. Polaronic effects obviously get stronger with increasing $\lambda$, eventually making the disorder distribution markedly bimodal. ${ }^{20}$ Correspondingly, the calculated behavior gradually approaches that obtained from a binary distribution of site energies, as realized for example in binary alloys ( $P_{0}^{B}(\epsilon)=[\delta(\epsilon-W)+\delta(\epsilon+W)] / 2$, gray lines). We note that in the case of binary disorder, the data in the explored range show little dependence on the electron-phonon interactions: the distribution of site energies is already bimodal in the absence of interactions, which is not altered by the presence of the deformable lattice.

\section{DISCUSSION}

We finally address the question of weak localization corrections, proving-based on an accurate comparison with existing experiments-our initial assertion that these are not the main cause of the observed Mooij correlations. Mott and Kaveh ${ }^{7}$ were the first to suggest that weak localization physics could lead to a change of sign of the TCR. Their results were subsequently used by Tsuei ${ }^{8}$ to argue that the behavior of TCR across different materials is nonuniversal, contrary to the initial claims by Mooij. ${ }^{5} \mathrm{~A}$ more careful analysis, however, shows that the formulation of ref. ${ }^{7}$ does indeed predict a universal TCR behavior, provided that one properly takes out all material-specific properties (a well-known analogy is that of phase transitions, which exhibit universal behavior only after the temperature is properly rescaled with the material-specific critical temperature $T_{\mathrm{c}}$ ). In particular, since the value of the "flat" resistivity $\rho^{*}$ varies from material to material, a meaningful scaling procedure-both for theory, and for the analysis of experimental data-should start by expressing resistivities in units of $\rho^{*}$. Following this idea, we were able to show that the weak localization formulas of refs. ${ }^{7,8}$ assume the following universal 
a

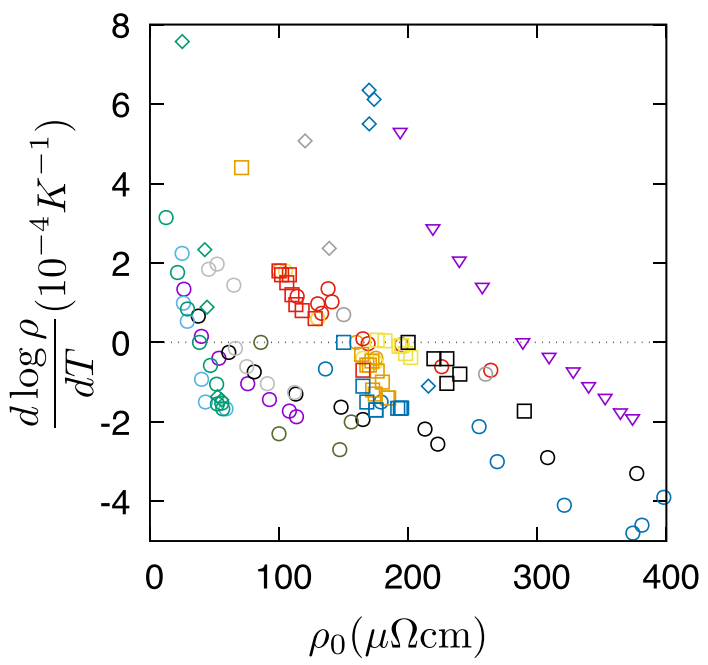

ErRhB [Dynes, Ref. (21)] AgCuAl [Mizutani, Ref. (22)] AgCuGe [Mizutani, Ref. (22)] AgCuMg [Mizutani, Ref. (22)] CaAl [Mizutani, Ref. (22)] NiP [Mizutani, Ref. (22)] NiPdP [Mizutani, Ref. (22)] PdCuP [Mizutani, Ref. (22)] CuSn [Mizutani, Ref. (22)] AgCuMg [Mizutani, Ref. (22)] CuAl [Mizutani, Ref. (22)] NiP [Naugle, Ref. (13)] b

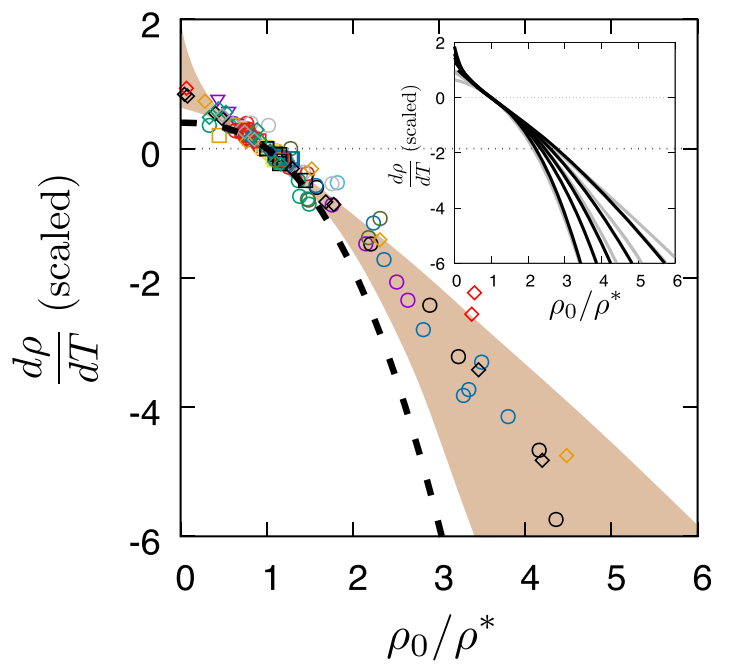

$\nabla \quad$ NiZr [Howson, Ref. (23)]

NiNb [Howson, Ref. (23)]

LaAl [Howson, Ref. (23)]

LaGa [Howson, Ref. (23)]

$\mathrm{NiP}$ [Howson, Ref. (23)]

GeSbTe [Siegrist, Ref. (24)]

FeSi [Sumiyama, Ref. (25)]

FeVAl [Feng, Ref. (26)]

NiNbZrH [Fukuhara, Ref. (27)]

CoMnCrSi [Aftab, Ref. (28)]

NiFeCr [Jin, Ref. (29)]

Fig. 3 Comparison with experimental data. a A collection of experimental data available in the literature, including data from ref. ${ }^{8}$ and more recent references. We have selected all series of data known to us which display a monotonic decrease of $\mathrm{d} \rho / \mathrm{d} T$ and a change of sign upon increasing randomness. b Same data, where each series has been rescaled as suggested in the text, allowing for comparison of disordered metals of a different chemical nature in the same plot. The inset reports the theoretical curves of Fig. $2 \mathrm{~b}$ scaled with the same procedure. Their span represents the whole range allowed by theory within the metallic phase, which is reported in the main plot as a shaded area. The dashed line is the weak localization theory of refs. ${ }^{78}$ triangles (from Dynes et al. ${ }^{22}$ ) and circles (from Mizutani ${ }^{23}$ and Naugle ${ }^{13}$ correspond to the data $^{24}$ already reported in Fig. 1 of ref. ${ }^{8}$ squares are from Howson and Gallagher. ${ }^{24}$ The remaining data are from Siegrist et al., ${ }^{25}$ Sumiyama et al., ${ }^{26}$ Feng, ${ }^{27}$ Fukuhara et al., 28 Aftab et al., ${ }^{29}$ and Jin et al. ${ }^{30}$ As the stoichiometry of the compounds can vary within each series, only the constituent elements are indicated

form (details in Sec. 2 of the SM file):

$\operatorname{TCR}\left(\rho_{0} / \rho^{*}\right)=\frac{2 C}{5}\left[1-\left(\rho_{0} / \rho^{*}\right)^{5 / 2}\right]$ (weak localization)

where $C$ is a prefactor which sets the slope of the curve TCR vs. $\left(\rho_{0} / \rho^{*}\right)$ at $\rho_{0}=\rho^{*}$, and which also depends on material-specific properties (e.g., the strength of the electron-phonon interactions and the carrier density).

Independently of the validity of Eq. (5), the scaling arguments provided above point to a better way of collecting the transport characteristics of different materials than reporting $\frac{\mathrm{dlog} \rho}{\mathrm{d} T}$ as a function of the zero-temperature intercept $\rho_{0}$ as is customarily done $^{5,8}$ (see Fig. 3a), by highlighting instead precisely those features of the TCR which are material independent. This is done in Fig. $3 b$, where we replot the experimental data for different compounds by rescaling the resistivities to the corresponding $\rho^{*}$, and then normalizing the slope to a common unit value. The first observation is that, in contrast with the original "Mooij plots", the data now show an almost complete collapse indicating that the proposed scaling idea is very powerful in rationalizing the experimental data of the whole class of disordered metals. The second observation is that the experiments markedly disagree with the weak localization predictions (dashed line), both quantitatively and qualitatively: not only do the data deviate appreciably from the behavior given by Eq. (5), but they also show significant scatter, (i.e., significant non-universality), incompatible with the weak localization scenario. In Fig. 3b, we also report our theoretical results based on the mechanism of polaronic disorder renormalization proposed in the present work, by applying an analogous scaling procedure to the data of Fig. $2 \mathrm{~b}$ : individual curves obtained for different values of the electron-phonon interaction strength and different models of disorder are reported in the inset; the full extent of the region allowed by theory is identified by the area spanned by all calculated curves, and is reported in the main panel (shaded area). As opposed to the weak localization scenario, in this case both the locus and scatter of data points are in excellent agreement with the data. Unscaled fits to the original experimental data set of ref. ${ }^{8}$ are shown in Fig. S3 of the SM file, illustrating the much better level of agreement achieved in that case by the present model as compared to weak localization theory.

The polaronic mechanism of strong disorder renormalization identified in this paper appears to provide a general and quantitatively accurate explanation for the mysterious hightemperature transport anomalies, ${ }^{6}$ which are commonly observed in strongly disordered metals. ${ }^{8}$ It is interesting to note that a very similar method of expanding around the MIR limit, again performed within the generalized DMFT setup, recently shed light $^{21}$ on another long-standing puzzle-that of the hightemperature "Bad Metal" behavior in doped Mott insulators. In that situation, which reflects strong electron-electron correlations in absence of disorder, one again finds linear-T resistivity, but its slope remains positive on both sides of the MIR limit. What happens when disorder and strong electron-electron interactions coexist remains a fascinating open question we reserve for future 
work. At any rate, we speculate that the mechanism illustrated here could very generally apply, leading to anomalous resistivity behavior whenever a "deformable" medium is present-atomic, electronic, or magnetic-that is able to locally respond to disorder.

\section{METHODS}

We study the following class of Hamiltonians combining disorder and electron-phonon interactions:

$$
H=-t \sum_{\langle i j\rangle, \sigma} c_{i, \sigma}^{\dagger} c_{j, \sigma}+\sum_{i, \sigma} \epsilon_{i} c_{i, \sigma}^{\dagger} c_{i, \sigma}-g \sum_{i, \sigma} c_{i, \sigma}^{\dagger} c_{i, \sigma} X_{i}+H_{p h}
$$

where $c_{i, \sigma}^{\dagger}\left(c_{i, \sigma}\right)$ are creation (annihilation) operators for electrons moving on a lattice of sites $i$ and spin $\sigma$ with transfer integral $t$. Disorder is characterized by a random distribution of atomic site energies $\epsilon_{i}$, denoted as $P_{0}\left(\epsilon_{i}\right)$. In addition to the random potential, the electrons interact locally with dispersionless phonons of frequency $\omega_{0}=\sqrt{K / M}$ described by $H_{p h}=\sum_{i} \frac{K X_{i}^{2}}{2}+\frac{P_{i}^{2}}{2 M^{2}}$. The strength of the electron-lattice coupling is measured by the dimensionless parameter $\lambda=g^{2} /(K D)$, with $D$ the halfbandwidth. The electron-lattice interaction term in Eq. (6) is solved using dynamical mean field theory (DMFT). Disorder is treated in the coherent potential approximation (CPA) (for full details, see the SM file, Sec. 1).

The general expressions in Eqs. (1) and (2) are obtained by expanding analytically the DMFT-CPA equations to lowest order in the lattice fluctuations, as described in Sec. 1.2 of the SM file. Equations (3) and (4) are obtained by further enforcing the classical approximation for phonons. The results reported in Figs. 2 and $3 \mathrm{~b}$ are obtained from the full numerical solution of the DMFT-CPA equations for classical phonons and considering two representative models of disorder: the uniform distribution $P_{0}(\epsilon)=\theta$ $\left(W^{2}-\epsilon^{2}\right) /(2 W)$ and the binary distribution $P^{B}{ }_{0}(\epsilon)=[\delta(\epsilon-W)+\delta(\epsilon+W)] / 2$, where in both cases the parameter $W$ measures the amount of randomness. From the local self-energy $\Sigma(\omega)$ obtained numerically, the electrical resistivity and the TCR are evaluated using the Kubo-Greenwood formula as described in Sec. 1.3 of the SM file.

\section{DATA AVAILABILITY}

The data sets generated during and/or analyzed during the current study are available from the corresponding author on reasonable request.

\section{ACKNOWLEDGEMENTS}

Work in Florida was supported by the NSF Grant No. DMR-1410132, and the National High Magnetic Field Laboratory through the NSF Cooperative Agreement No. DMR1157490 and the State of Florida. D.D.S. acknowledges the German Research Foundation (DFG-SFB 1170), the ERC-StG-336012-Thomale-TOPOLECTRICS, and the Gauss Centre for Supercomputing e.V. (www.gauss-centre.eu) for funding this project by providing computing time on the GCS Supercomputer SuperMUC at Leibniz Supercomputing Centre (LRZ, www.Irz.de).

\section{AUTHOR CONTRIBUTIONS}

S.C., V.D., and S.F. led the project and co-wrote the paper. D.D.S. and S.C. implemented the method.

\section{ADDITIONAL INFORMATION}

Supplementary information accompanies the paper on the npj Quantum Materials website (https://doi.org/10.1038/s41535-018-0119-y).

Competing interests: The authors declare no competing interests.

Publisher's note: Springer Nature remains neutral with regard to jurisdictional claims in published maps and institutional affiliations.

\section{REFERENCES}

1. Fisk, Z. \& Webb, G. W. Saturation of the high-temperature normal-state electrical resistivity of superconductors. Phys. Rev. Lett. 36, 1084-1086 (1976).

2. Hussey, N., Takenaka, K. \& Takagi, H. Universality of the Mott-loffe-Regel limit in metals. Philos. Mag. 84, 2847-2864 (2004).
3. Calandra, M. \& Gunnarsson, O. Electrical resistivity at large temperatures: saturation and lack thereof. Phys. Rev. B 66, 205105 (2002).

4. Gunnarsson, O., Calandra, M. \& Han, J. E. Colloquium: saturation of electrical resistivity. Rev. Mod. Phys. 75, 1085-1099 (2003).

5. Mooij, J. H. Electrical conduction in concentrated disordered transition metal alloys. Phys. Status Solidi 17, 521-530 (1973).

6. Lee, P. A. \& Ramakrishnan, T. V. Disordered electronic systems. Rev. Mod. Phys. 57, 287-337 (1985).

7. Kaveh, M. \& Mott, N. F. Universal dependences of the conductivity of metallic disordered systems on temperature, magnetic field and frequency. J. Phys. C 15, L707-L716 (1982).

8. Tsuei, C. C. Nonuniversality of the Mooij correlation - the temperature coefficient of electrical resistivity of disordered metals. Phys. Rev. Lett. 57, 1943-1946 (1986).

9. Imry, Y. Possible role of incipient Anderson localization in the resistivities of highly disordered metals. Phys. Rev. Lett. 44, 469-471 (1980).

10. Gantmakher, V. F. Mooij rule and weak localization. JETP Lett. 94, 626-628 (2011).

11. Anderson, P. W. Absence of diffusion in certain random lattices. Phys. Rev. 109, 1492-1505 (1958).

12. Nagel, S. R. Metallic glasses. Adv. Chem. Phys. 51, 227-275 (1982).

13. Naugle, D. G. Electron transport in amorphous metals. J. Phys. Chem. Solids $\mathbf{4 5 ,}$ 367-388 (1984).

14. Anderson, P. W. Effect of franck-condon displacements on the mobility edge and the energy gap in disordered materials. Nature 235, 163-165 (1972).

15. Di Sante, D., Fratini, S., Dobrosavljević, V. \& Ciuchi, S. Disorder-driven metal-insulator transitions in deformable lattices. Phys. Rev. Lett. 118, 036602 (2017).

16. Millis, A. J., Hu, J. \& Das Sarma, S. Resistivity saturation revisited: results from a dynamical mean field theory. Phys. Rev. Lett. 82, 2354-2357 (1999).

17. Galitski, V. M., Adam, S. \& Das Sarma, S. Statistics of random voltage fluctuations and the low-density residual conductivity of graphene. Phys. Rev. B 76, 245405 (2007).

18. Logan, D. E. \& Wolynes, P. G. Dephasing and Anderson localization in topologically disordered systems. Phys. Rev. B 36, 4135-4147 (1987).

19. Girvin, S. M. \& Jonson, M. Dynamical electron-phonon interaction and conductivity in strongly disordered metal alloys. Phys. Rev. B 22, 3583-3597 (1980).

20. Millis, A. J., Mueller, R. \& Shraiman, B. I. Fermi-liquid-to-polaron crossover. I. general results. Phys. Rev. B 54, 5389-5404 (1996).

21. Vučičević, J., Tanasković, D., Rozenberg, M. \& Dobrosavljević, V. Bad-metal behavior reveals Mott quantum criticality in doped Hubbard models. Phys. Rev. Lett. 114, 246402 (2015).

22. Dynes, R. C., Rowell, J. M. \& Schmidt, P. H. Ternary Superconductors (eds Shenoy, G. K., Dunlap, B. D. \& Fradin, F. Y.) (North-Holland, Amsterdam, 1981).

23. Mizutani, U. Electronic structure of metallic glasses. Prog. Mater. Sci. 28, 97-228 (1983).

24. Howson, M. A. \& Gallagher, B. L. Disorder-induced localization in crystalline phase-change materials. Phys. Rep. 170, 265-324 (1988).

25. Siegrist, T. et al. Disorder-induced localization in crystalline phase-change materials. Nat. Mater. 10, 202-208 (2011).

26. Sumiyama, K. et al. Electric and magnetic evolution in sputter-deposited $\mathrm{Fe}_{x} \mathrm{Si}_{x}$ alloy films. Mater. Trans. 57, 907-912 (2016).

27. Feng, Y. et al. Physical properties of heusler-like Fe 2 VAl. Phys. Rev. B 63, 165109, (2001).

28. Fukuhara, M., Gangli, C., Matsubayashi, K. \& Uwatoko, Y. Pressure-induced positive electrical resistivity coefficient in Ni-Nb-Zr-H glassy alloy. Appl. Phys. Lett. 100, 253114 (2012).

29. Aftab, M., Hassnain Jaffari, G., Hasanain, S. K., Ali Abbas, T. \& Ismat Shah, S. Magnetic and transport properties of $\mathrm{Co}_{2} \mathrm{Mn}_{1-x} \mathrm{Cr}_{x} \mathrm{Si}$ Heusler alloy thin films. J. Appl. Phys. 114, 103903 (2013).

30. Jin, K. et al. Tailoring the physical properties of Ni-based single-phase equiatomic alloys by modifying the chemical complexity. Sci. Rep. 6, 20159 (2016).

Open Access This article is licensed under a Creative Commons Attribution 4.0 International License, which permits use, sharing, adaptation, distribution and reproduction in any medium or format, as long as you give appropriate credit to the original author(s) and the source, provide a link to the Creative Commons license, and indicate if changes were made. The images or other third party material in this article are included in the article's Creative Commons license, unless indicated otherwise in a credit line to the material. If material is not included in the article's Creative Commons license and your intended use is not permitted by statutory regulation or exceeds the permitted use, you will need to obtain permission directly from the copyright holder. To view a copy of this license, visit http://creativecommons. org/licenses/by/4.0/.

(c) The Author(s) 2018 